\title{
EDITORIAL
}

\section{Repeated echocardiography after the diagnosis of endocarditis: too much of a good thing?}

\author{
C H Cabell, V G Fowler Jr
}

Heart 2004;90:975-976. doi: 10.1136/hrt.2003.031328

\begin{abstract}
Although echocardiography is seen as the technology of choice for the diagnosis of infective endocarditis, it is being increasingly overused in clinical scenarios with a low pretest probability of disease
\end{abstract}

See end of article for authors' affiliations

Correspondence to: Dr Christopher H Cabell, Box 3850, Duke University Medical Center, Durham, NC 27710, USA; chris. cabell@duke.edu
S nce the advent of two dimensional transthoracic echocardiography (TTE) in the 1970s and high frequency transoesophageal echocardiography (TOE) imaging in the 1980s, echocardiography has become a standard diagnostic tool in patients with suspected infective endocarditis (IE). It is now well established that echocardiography is a technology of choice for the diagnosis of IE, ${ }^{1}$ and that echocardiography can detect cardiac involvement in a significant proportion of patients with clinically occult IE. ${ }^{23}$ Because IE is a lethal infection that can be difficult to diagnose clinically, clinicians who care for patients at risk for IE often have a low threshold for employing echocardiography. This clinical practice has several implications. While echocardiography can often provide a rapid diagnosis, its optimal use is predicated on the appropriate pre-test probability of disease. ${ }^{45} \mathrm{By}$ contrast, echocardiography is increasingly overused in clinical scenarios with a low $(<2-3 \%)$ pre-test probability of disease, where its diagnostic utility diminishes. ${ }^{67}$

\section{DIAGNOSTIC YIELD}

It is in this light that Vieira and colleagues, ${ }^{8}$ in a study reported in this issue of Heart, have asked an important question: "What is the diagnostic yield of repeated echocardiograms in patients with suspected IE?" Over a three year period, they evaluated 262 patients with suspected IE referred for echocardiography. They found that repeat echocardiography was frequent: TTEs were repeated at least once in 192 (72.2\%) patients while TOEs were repeated in 49 (18.4\%) of patients. The average number of TTE examinations was 2.4 , but six patients had at least six TTEs. In a similar fashion, the mean number of TOE examinations was 1.7, although four patients had at least four TOEs and one patient had five. The authors found that while repeated echocardiograms were occasionally helpful, no additional diagnostic information was provided after the second or third echocardiogram (TTE or TOE).

Although this information is helpful in understanding the limitations of excessive imaging, it is unclear how many of the 262 patients had both TTE and TOE examinations and how these findings affected subsequent evaluation. In addition, the clinical setting of a large university cardiology hospital with a high percentage of patients with prosthetic valves and intracardiac devices may have skewed the findings toward an increase in the use of echocardiography. Finally, the aggressive use of imaging may reflect a high clinical suspicion for underlying IE, as most of these patients were referred to the university based cardiology hospital in which the study took place with positive blood cultures and other suggestive findings.

\section{ECHO OVERUSED}

These limitations notwithstanding, it is evident from this and previous studies that imaging technologies such as echocardiography can be overused in certain clinical scenarios such as the evaluation of suspected endocarditis. For instance, Kurupuu and colleagues have shown that $53 \%$ of echocardiograms could be avoided without loss of diagnostic accuracy by using a simple algorithm in patients with a low pre-test probability of disease. ${ }^{6}$ In addition, Greaves and colleagues have shown that the collective absence of five simple clinical criteria indicated a zero probability of a TTE showing evidence of endocarditis. ${ }^{7}$ Collectively, these studies have shown that in patients with very low pre-test probability of disease that echocardiography may be avoided without the loss of diagnostic accuracy.

Although current guidelines highlight the use of TTE and/or TOE imaging depending on the clinical scenario, studies have shown that a strategy of TOE imaging is the most cost effective in the majority of clinical situations. For example, Heidenreich and colleagues have show that in suspected endocarditis a diagnostic strategy that focuses on TOE as the initial imaging modality is more cost effective than a staged procedure with TTE and is a dominant strategy over empiric antibiotic treatment alone. ${ }^{5}$

For a variety of reasons it is important to limit the indiscriminate use of diagnostic technologies. From a safety standpoint, low risk procedures such as TOE may have an unacceptable risk:benefit ratio as the diagnostic yield approaches zero. In addition, in the era of rapidly

Abbreviations: IE, infective endocarditis; TOE, transoesophageal echocardiography; TTE, transthoracic echocardiography 
rising medical costs, excessive procedures lead to substantial financial waste. For example, in the USA it has been estimated that the appropriate use of TOE to establish the length of treatment in catheter associated Staphylococcus aureus bacteraemia could save over $\$ 120$ million annually. ${ }^{10}$

\section{PRE-TEST PROBABILITY OF DISEASE}

It is clear from the work by Vieira and colleagues, ${ }^{8}$ as well as previous studies, that the real issue in evaluating patients with suspected endocarditis is the pre-test probability of disease. In any given patient at risk for IE, such as patients with prosthetic valves, this pre-test probability may be different for different microorganisms. Studies are needed that focus attention on understanding which clinical characteristics are the most important in predicting risk for the subsequent development of IE. From these studies, scoring systems could be developed that help clinicians at the bedside improve their precision in developing the appropriate pre-test probability for disease. A scoring system such as this could be tested prospectively, validated, and then disseminated into clinical practice by existing guideline committees. Until such data become available, it will be very difficult to limit the indiscriminate use of expensive diagnostic modalities by our technology driven society.

\section{ACKNOWLEDGEMENT}

This work was supported in part by National Institutes of Health grants HL70861 (CHC) and R01AI59111 (VGF)

\section{Authors' affiliations}

C H Cabell, V G Fowler, Department of Medicine, Duke University School of Medicine, and the Duke Clinical Research Institute, Durham, North Carolina, USA

\section{REFERENCES}

1 Durack DT, Lukes AS, Bright DK. New criteria for diagnosis of infective endocarditis: Utilization of specific echocardiographic findings. Am J Med 1994;96:200-9.

2 Fowler VG, Li J, Corey GR, et al. Role of echocardiography in evaluation of patients with Staphylococcus aureus bacteremia. J Am Coll Cardiol 1997;30:1072-8

3 Pedersen WR, Walker M, Olson JD, et al. Value of transesophageal echocardiography as an adjunct to transthoracic echocardiography in evaluation of native and prosthetic valve endocarditis. Chest 1991;100:351-6.

4 Lindner JR, Case AC, Dent JM, et al. Diagnostic value of echocardiography in suspected endocarditis. Circulation 1996;93:730-6.

5 Heidenreich P, Masoudi F, Maini B, et al. Echocardiography in patients with suspected endocarditis: a cost effectiveness analysis. Am J Med 1999; 107:198-208

6 Kuruppu JC, Corretti M, Mackowiak P, et al. Overuse of transthoracic echocardiography in the diagnosis of native valve endocarditis. Arch Intern Med 2002;162:1715-20.

7 Greaves K, Patel A, Celermajer D. Clinical criteria and the appropriate use of transthoracic echocardiography for the exclusion of infective endocarditis. Heart 2003;89:273-5

8 Vieira MLC, Grinberg M, Pomerantzeff PMA, et al. Repeated echocardiographic examinations of patients with suspected infective endocarditis. Heart 2004;90:1020-4

9 Bayer AS, Bolger AF, Taubert KA, et al. Diagnosis and management of infective endocarditis and its complications. Circulation 1998;98:2936-48.

10 Rosen AB, Fowler VG, Corey GR, et al. Cost-effectiveness of transesophageal echocardiography to determine the duration of therapy for intravascular catheter-associated Staphylococcus aureus bacteremia. Ann Intern Med $1999 ; 130: 810-20$.

\section{IMAGES IN CARDIOLOGY}

\section{5 years follow up of pacing in a toddler}

W 1977, a 30 month old female child with congenital complete heart block and recurrent Stokes Adams attacks underwent single chamber (VVI) permanent pacemaker implantation in the anterior abdominal wall with tunnelled epicardial pacing lead. Due to local infection the pacing system had to be removed and the lead cut (abdominal lead 3, panel A). The next site chosen for placement of the generator (PM-2) was intrathoracic, in the left retropleural space by lateral thoracotomy. During the placement of this left ventricular epicardial lead (lead 1), the cardiac end of the initial lead had been cut close to the heart (thoracic lead 3). After 14 years, the same author placed a transvenous single pacing system (PM-2) with endocardial right ventricular lead (lead 2). Panel B shows the chest $x$ ray in postero-anterior (PA) view. Now aged 28 years, the patient is married with two healthy children who were delivered uneventfully. Generator change for battery depletion is due in six months.

Pacing in infancy and childhood was a challenging task in the 1960s and '70s. Large generators (weighing about $200 \mathrm{~g}$ ), a greater risk of perforation and secondary infection, frequent

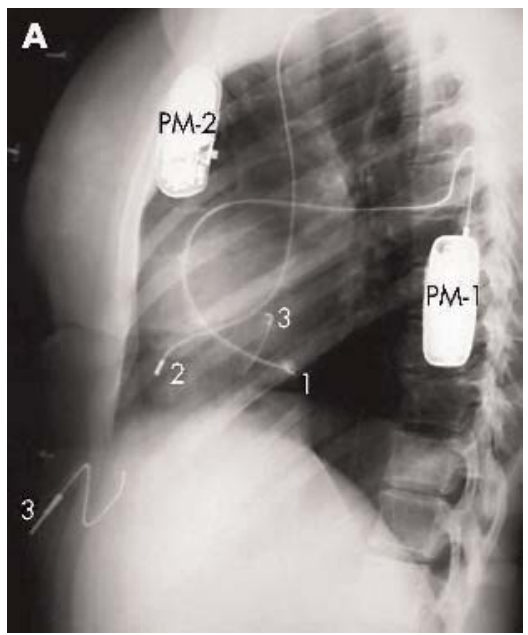

Lateral view, showing the location of both implanted pacemakers and their respective leads, which are easily traced.

generator replacement due to short battery life, and the growth of the child which could dislodge lead systems and alter pacing parameters, were only a few of the issues that had to be tackled. Due to lack of space in the subcutaneous tissue of the chest wall, generators were

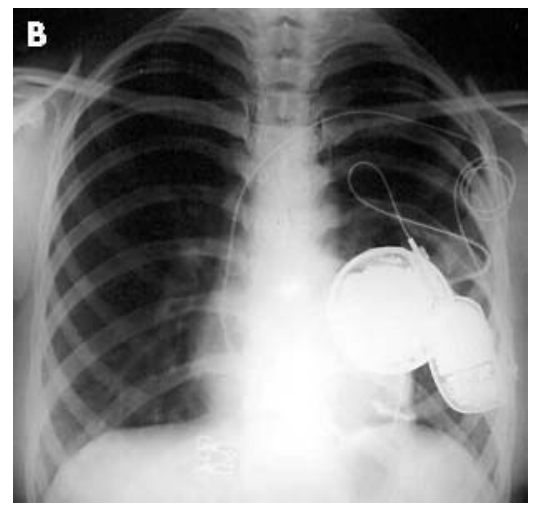

PA view, where it is difficult to follow the pacers and their lead systems due to their superimposition.

implanted surgically in the abdomen or retropleural space. Newer pacing techniques and the reduction in generator size (to about $20 \mathrm{~g}$ ) has resulted in much easier and safer pacing outcomes today.

A Chockalingam S Dorairajan V Chockalingam drcanands@yahoo.co.in 